\title{
Spectroscopy in the vacuum-ultraviolet
}

To the Editor - The recently presented results in Nature Photonics on Fourier transform spectroscopy (FTS) in the vacuum-ultraviolet (VUV) range are truly impressive ${ }^{1}$. With a resolution much greater than what can be attained using grating spectrometers and the innate advantages of simultaneous wavelength accuracy and broad tunability, this technological development is of great importance and it will certainly open up new fields of research. However, the implemented design is limited to operation at wavelengths longer than $40 \mathrm{~nm}$, owing to miniscule reflectivities at large glancing angles of incidence for the beamsplitter. To extend FTS to even shorter wavelengths, reflection at grazing incidence is necessary. The experimental challenge is therefore to manufacture and use appropriate wavefront-dividing grazingincidence beamsplitters. De Oliveria et al. ${ }^{1}$ state that FTS using amplitude-dividing beamsplitters has so far been limited to wavelengths of larger than $140 \mathrm{~nm}$, and that attempts to use wavefront-dividing beamsplitters in a Mach-Zehnder-type design ${ }^{2}$ have been unsuccessful.

Here we would like to point out that a modified Mach-Zehnder Fourier transform spectrometer ${ }^{3}$, similar in conceptual design to the one described in ref. 2 , has been built and tested with $\mathrm{He}$ I and He II radiation ${ }^{4}$, and was also shown to be capable of reaching wavelengths as short as $22.5 \mathrm{~nm}$ (55 eV; Fig. 1). Although this spectrometer is indeed based on wavefrontdividing beamsplitters as suggested in ref. 2, the scanning length is much shorter than in ref. 1 and hence it cannot yet compete in terms of energy resolution. Wavefront-dividing beamsplitters based on the microstructuring of silicon nitride membranes were recently developed ${ }^{5}$, which may extend the FTS technique to even shorter wavelengths.

It should be noted that large-aperture wavefront-dividing beamsplitters ${ }^{4}$ make it possible to use the FTS technique not only for absorption studies (as in ref. 1), but also for the analysis of secondary photons. This will not only increase the number of applications of this technique but will also facilitate resonant inelastic soft-X-ray/VUV inelastic scattering (RIXS) experiments with unprecedented energy resolution. In this energy range, the behaviour of double excitations of the helium atom

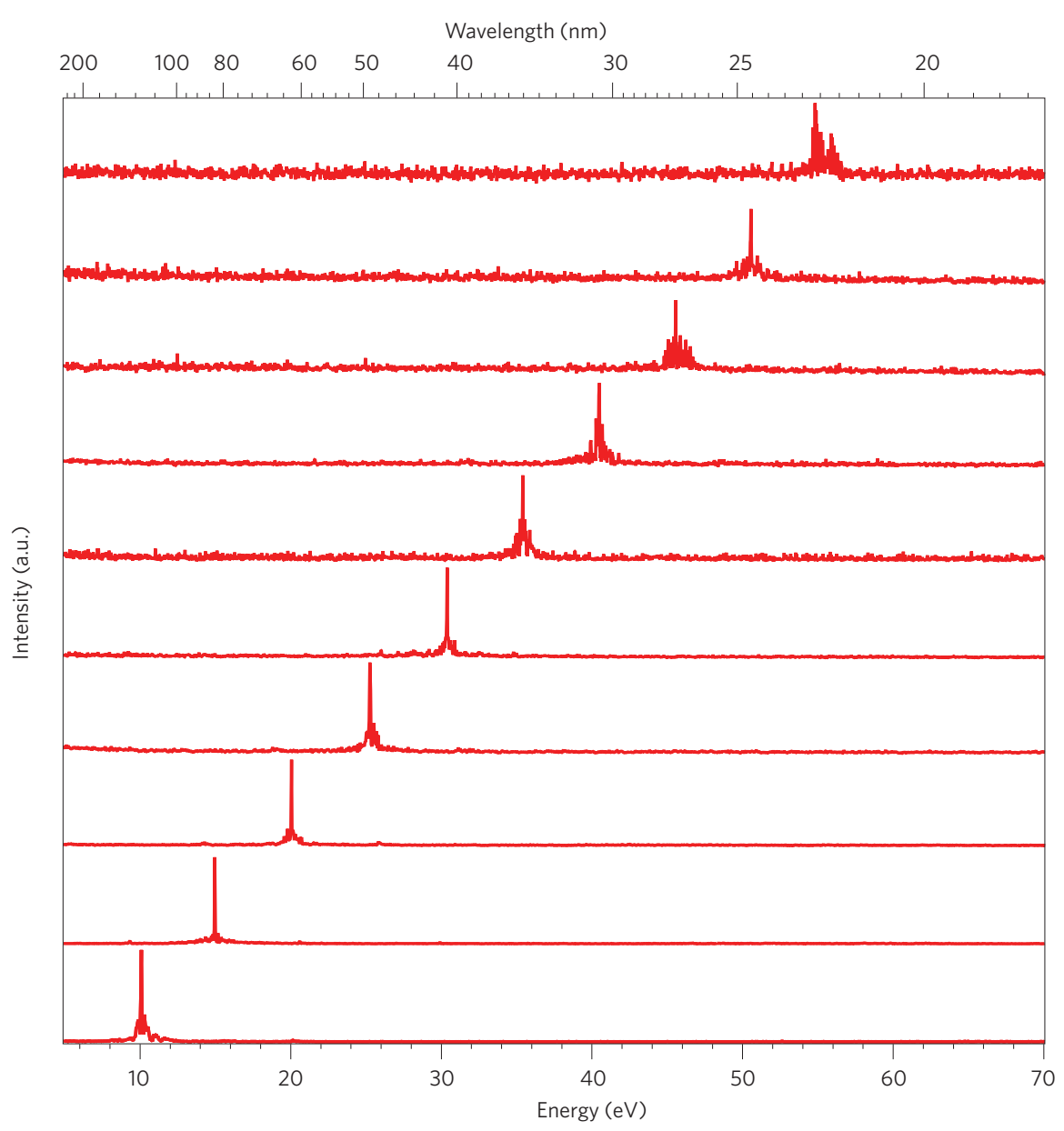

Figure 1 | Fast Fourier transform spectra of interferograms measured with a modified grazing-incidence Mach-Zehnder interferometer at the NIM beamline I3 at MAX-lab, Lund, Sweden. The interferometer probes the monochromatic beam to test the system's ability to perform FTS at various VUV energies. The energy scale was set using a $650 \mathrm{~nm}$ diode laser running parallel to the VUV beam through the interferometer.

(the prototype two-electron system) continues to challenge state-of-the-art atomic physics theory. Moreover, the inner shells of transition metal M-edges come within reach at such energies, allowing for RIXS investigations of the fundamental excitations in correlated compounds ${ }^{6}$, including most high-temperature superconductors. The ability to achieve high energy resolution is essential for such studies. We are looking forward to further developments in this exciting new field.

References

1. de Oliveria, N. et al. Nature Photon. 5, 149-153 (2011)

2. Howells, M. R. et al. Nucl. Inst. Meth. Phys. Res. A 347, 182-191 (1994)
3. Yin, H., Wang, M., Ström, M. \& Nordgren, J. Nucl. Inst. Meth. Phys. Res. A 451, 529-539 (2000)

4. Agåker, M. et al. Nucl. Inst. Meth Phys. Res. A 601, 213-219 (2009)

5. Wilcox, J., White, V., Shcheglov, K. \& Kowalcyzk, R. Proc. SPIE 7802, 780206 (2010)

6. Ament, L. J. P., van Veenendaal, M., Devereaux, T., Hill, J. P. \& van den Brink, J. Preprint at http://arxiv.org/abs/1009.3630 (2010)

M. Agåker ${ }^{1 *}$, J. Andersson'1, J. C. Englund', J. Rausch ${ }^{2}$, J.-E. Rubensson ${ }^{1}$ and J. Nordgren ${ }^{1}$ ${ }^{1}$ Department of physics and astronomy, Uppsala University, Box 516 75120, Uppsala, Sweden. ${ }^{2}$ Institut für Atom- und Molekülphysik, Justus Liebig University, 35392, Giessen, Germany.

*e-mail: marcus.agaker@fysik.uu.se 